\title{
HIV vaccine failure due to induction of immune suppressors?
}

An effective therapeutic and/or prophylactic vaccine against HIV would be the ultimate weapon against the global AIDS pandemic. However, although it has been shown that vaccine-induced $\mathrm{T}$ cell responses can control HIV replication, the formulation of effective vaccines has been exceptionally challenging. Now, writing in the Journal of Clinical Investigation, Sui, Berzofsky and colleagues report an unexpected stumbling block in anti-HIV vaccine development: they show that vaccines can potentially induce suppressor cells and thereby dampen any specific immune responses.

This finding was based on a serendipitous discovery in a vaccine study in a non-human primate model of HIV infection using the simian

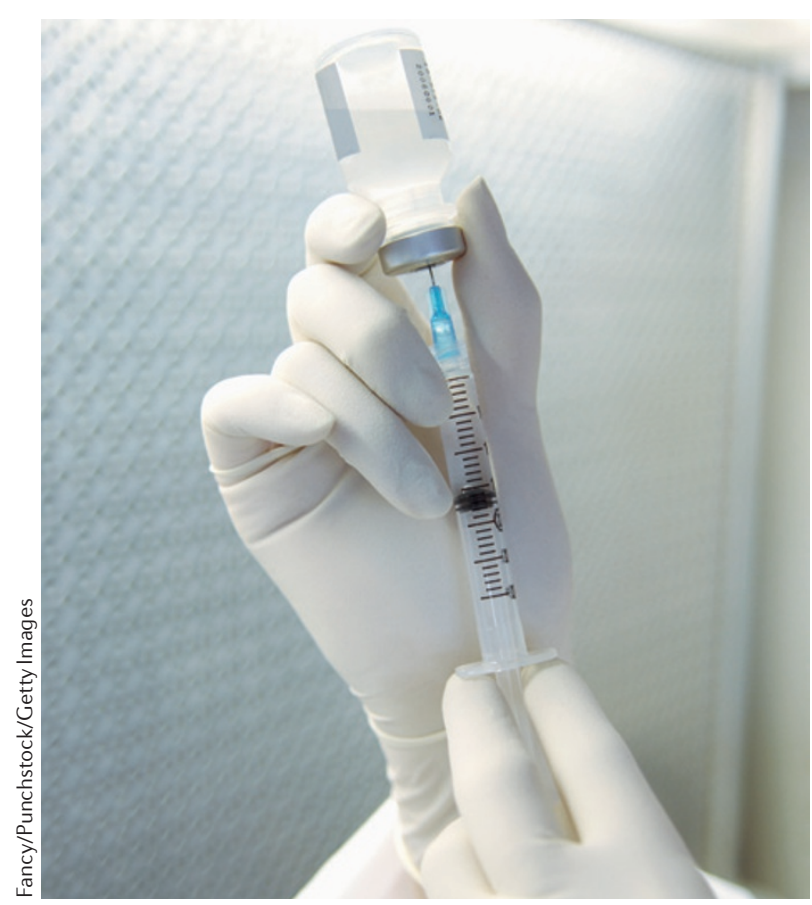

immunodeficiency virus (SIV). To explore the potential of $\mathrm{T}$ cell-based HIV/SIV vaccines, the authors used a peptide-prime/modified vaccinia Ankara (MVA) boost vaccine with Toll-like receptor 2 (TLR2), TLR3 and TLR9 agonists as well as interleukin-15 (IL-15) as base adjuvants. a-GalCer, a natural killer T (NKT) cell agonist, as well as B7-DC-Ig, an engineered modulator of the checkpoint inhibitor programmed cell death protein 1 (PD1; which suppresses $\mathrm{T}$ cell activation), were tested as additional adjuvants. The vaccine and different combinations of adjuvants were applied intrarectally, and control groups consisted of animals that were either left untreated (naive) or received all the adjuvants but not the actual vaccine. The animals were then repeatedly challenged with the virus until all animals were infected. During the acute infection, none of the vaccinated groups showed lower viral loads (VLs) than the naive control animals. Surprisingly though, during both the acute and chronic phase, the control group that received adjuvant only showed a substantial VL decrease compared to vaccinated animals that were given the same adjuvants, indicating that the vaccine reduced the beneficial effect of the adjuvants. This effect was highly significant in animals with one particular major histocompatibility complex (MHC) class I allele, and in vivo lymphocyte depletion studies showed that the antiviral response was largely mediated by CD8 ${ }^{+} \mathrm{T}$ cells.

The authors speculated that the negative effect of the vaccine may be due to the induction of target cells for infection (virus-specific CD $4^{+} \mathrm{T}$ cells not only provide help to $\mathrm{CD} 8^{+} \mathrm{T}$ cell responses but are also preferentially infected upon viral challenge) and/or that the vaccine may induce suppressive regulatory cells. Examining the immune cells in the post-vaccination mucosal tissues, they indeed found that animals that received the vaccine had significantly higher levels of $\mathrm{CCR} 5^{+} \mathrm{Ki} 67^{+} \mathrm{CD} 4^{+} \mathrm{T}$ cells. These serve as viral targets in colorectal tissues and their frequency correlated with VLs in the early stages of infection. Moreover, myeloid-derived suppressor cells (MDSCs), which are known to suppress $\mathrm{T}$ cell responses in patients with cancer and in HIV-1infected individuals, were increased in vaccinated animals but not in the adjuvant-only group. Further in vitro and in vivo analysis established a positive correlation between VLs in the chronic phase of disease and the frequency of MDSCs, and demonstrated that vaccine-induced MDSCs can suppress CD8 ${ }^{+} \mathrm{T}$ cell responses. Moreover, it was found that transforming growth factor- $\beta$ (TGF $\beta$ ), which is known to have a role in the induction of MDSCs, was significantly elevated in the plasma of animals that received the vaccine.

The authors suggest a model in which MDSCs that are induced by the vaccination suppress protective $\mathrm{CD} 8^{+}$ $\mathrm{T}$ cell responses, and conclude that the prevention of MDSC expansion (for example, by inhibition of TGF $\beta$ ) may be critical to achieving vaccine efficacy against viruses such as HIV. Alexandra Flemming

ORIGINAL RESEARCH PAPER Sui Y. et al. Vaccine-induced myeloid cell population dampens protective immunity to SIV.J. Clin. Invest. 124, 2538-2549 (2014) 